\title{
Targeting tumour microenvironment, a FAKtual challenge in pancreatic cancer
}

\author{
Ezequiel J Tolosa, Martín E Fernández-Zapico
}

In the past two decades, investigators have turned their attention to the development of new approaches to inhibit the aggressive behaviour of the tumour microenvironment (TME) of pancreatic ductal adenocarcinoma (PDAC), ${ }^{1-7}$ a dismal disease predicted to be the second leading cause of cancer death by 2030 . However, complete genetic or pharmacological ablation of stroma results in more aggressive tumours. Furthermore, targeting the TME in patients with PDAC has grown controversial due to failure of clinical trials to improve patient survival. ${ }^{8} 9$ Together, these findings highlight the need to increase our understanding of the role of TME components (cellular and noncellular) and their interplay in PDAC pathogenesis. This would better inform development of new treatment approaches not aimed at total stromal depletion, but rather TME reprogramming to 'activate' its antitumoural functions or to facilitate sensitivity to specific targeted therapies. In Gut, Jiang and colleagues elegantly report a novel therapeutic approach through partial stromal depletion, employing clinically available focal adhesion kinase (FAK) inhibitors to sensitise PDAC to signal transducer and activator of transcription 3 (STAT3) inhibitors. This opens new avenues to develop targeted therapy approaches for patients with PDAC having tumour stromal depletion following initial treatment.

Specifically, Jiang et al describe a FAK inhibition resistance mechanism associated with depletion of $\alpha$-smooth muscle actin ( $\alpha$-SMA)-positive cancer-associated fibroblasts (CAFs) and subsequent decreased collagen deposition. ${ }^{10}$ A previous publication by this same group reported promising findings that abrogation of hyperactivated FAK signalling in PDAC models using VS-4718, an inhibitor currently in clinical trials for patients with PDAC (clinical trials: NCT02546531, NCT03727880 and NCT02758587), diminished stromal density, thereby increasing the tumour response to chemotherapy and

Schulze Center for Novel Therapeutics, Mayo Clinic, Rochester, Minnesota, USA

Correspondence to Dr Martín E Fernández-Zapico, Schulze for Novel Therapeutics, Mayo Clinic, Rochester, MN 55905, USA; fernandezzapico.martin@mayo.edu immunotherapy. However, some PDACs exhibited a period of disease stabilisation followed by tumour resistance to VS-4718 and rapid tumour progression. ${ }^{11}$ Using a combination of genetically engineered mouse models and a well-executed set of in vitro assays, the authors identified STAT3 signalling hyperactivation as a compensatory survival mechanism following prolonged FAK inhibitor treatment. The authors demonstrated increased expression levels of phosphorylatedSTAT3 (pSTAT3) in tumours with limited response to FAK inhibition and shorter survival in mice. Using well-established PDAC mouse models including KPC ( $p 48$ Cre, LSL-Kras $G^{12 D / w t}$ and $\left.p 53^{\text {flox/wt }}\right)$, KPPC (p48-Cre, LSL-Kras ${ }^{G 12 D / w t}$ and $p 53^{\text {flox/ }}$ $\left.{ }^{f l o x}\right)$ and syngeneic transplantable models, the investigators found that survival with VS-4718 FAK inhibitor treatment was inversely correlated with tumour pSTAT3 levels. Further, using RNA interferencebased approaches in vitro and in vivo, the authors demonstrated STAT3 signalling as the primary modulator of resistance to FAK inhibition.

Further analysis of this phenomenon revealed that prolonged FAK inhibitor treatment led to a reduction in transforming growth factor beta 1 (TGF- $\beta 1$ ) and SMAD family member 3 (SMAD3) signalling in PDAC TME. This effect was accompanied by decreased $\alpha$-SMApositive CAFs and collagen density. Treatment of KPPC animals with a TGF- $\beta$-neutralising antibody mimicked the phenotype of prolonged FAK inhibition, promoting upregulation of pSTAT3 in PDAC tissues. This suggested that stromal TGF- $\beta 1$-mediated antagonism of STAT3 activation prevented tumour resistance. The authors implicated the TGF- $\beta 1 /$ SMAD3 signalling axis in this phenomenon without significant involvement of other TGF- $\beta$ or bone morphogenetic protein (BMP) receptors or other TGF- $\beta$ receptors ligands. Moreover, the authors showed that the expression of TGF- $\beta 1$ in the TME and activation of SMAD3 in PDAC cells counteracted the activation of STAT3 in a time-dependent and dose-dependent manner. In addition, genetic knockdown of STAT3 sensitised PDAC cells to FAK inhibition. Interestingly, Jiang et al also showed that
STAT3-mediated FAK inhibitor resistance could be overcome with a dual pharmacological inhibition of FAK and Janus kinase (JAK)/STAT3 pathways suppressed PDAC progression. FAK inhibitor combination with ruxolitinib (JAK1/2 inhibitor) or Stattic (STAT3 inhibitor) resulted in synergistic reduction in PDAC cell proliferation in vitro and tumour growth in a syngeneic and KPPC models, suggesting that blocking JAK/STAT3 signalling favours PDAC responsiveness to FAK inhibition. $^{10}$

In summary, these findings provide a new framework for the development of new treatment strategies, taking advantage of the interplay between components of TME in PDAC. Jiang and colleagues proposed a new STAT inhibitor-based treatment for cases with significant stromal depletion. As opposed to complete abrogation of the PDAC TME, this represents a suitable alternative strategy as the field continues to develop a better understanding of the dynamic relationship between tumour and TME elements. Such clarity is necessary to develop new strategies to tactically and dynamically modulate the TME to enhance PDAC therapy.

Acknowledgements We thank Dr Ryan Carr for critically reading the manuscript and providing helpful advice.

Contributors Both authors have equally contributed to the conceptualisation and writing of the manuscript.

Funding This work was supported by National Cancer Institute CA136526.

Competing interests None declared.

Patient consent for publication Not required.

Provenance and peer review Commissioned; internally peer reviewed.

\section{0 OPEN ACCESS}

Open access This is an open access article distributed in accordance with the Creative Commons Attribution Non Commercial (CC BY-NC 4.0) license, which permits others to distribute, remix, adapt, build upon this work non-commercially, and license their derivative works on different terms, provided the original work is properly cited, appropriate credit is given, any changes made indicated, and the use is non-commercial. See: http:// creativecommons.org/licenses/by-nc/4.0/.

(C) Author(s) (or their employer(s)) 2020. Re-use permitted under CC BY-NC. No commercial re-use. See rights and permissions. Published by BMJ.

\section{(D) Check for updates}

To cite Tolosa EJ, Fernández-Zapico ME. Gut 2020;69:1-2.

Received 8 July 2019

Revised 19 September 2019

Accepted 23 September 2019 
Published Online First 3 October 2019

\section{CLinked}

- http://dx.doi.org/10.1136/gutjnl-2018-317424

Gut 2020;69:1-2.

doi:10.1136/gutjnl-2019-318962

ORCID ID

Martín E Fernández-Zapico http://orcid.org/0000-00028089-3907

\section{REFERENCES}

1 Aran D, Sirota M, Butte AJ. Systematic pancancer analysis of tumour purity. Nat Commun 2015;6:8971.
2 Erkan M, Adler G, Apte MV, et al. StellaTUM: current consensus and discussion on pancreatic stellate cell research. Gut 2012;61:172-8

3 Erkan M, Reiser-Erkan C, W. Michalski C, et al. The impact of the activated stroma on pancreatic ductal adenocarcinoma biology and therapy resistance. Curr Mol Med 2012;12:288-303.

4 Hwang RF, Moore T, Arumugam T, et al. CancerAssociated stromal fibroblasts promote pancreatic tumor progression. Cancer Res 2008;68:918-26.

5 Mantoni TS, Lunardi S, Al-Assar 0, et al. Pancreatic stellate cells radioprotect pancreatic cancer cells through $\beta 1$-integrin signaling. Cancer Res 2011;71:3453-8.

6 Olive KP, Jacobetz MA, Davidson CJ, et al. Inhibition of Hedgehog signaling enhances delivery of chemotherapy in a mouse model of pancreatic cancer. Science 2009;324:1457-61.

7 Catenacci DVT, Junttila MR, Karrison T, et al. Randomized phase Ib/ll study of gemcitabine plus placebo or vismodegib, a hedgehog pathway inhibitor in patients with metastatic pancreatic cancer. JCO 2015:33:4284-92.

8 Özdemir BC, Pentcheva-Hoang T, Carstens JL, et al. Depletion of carcinoma-associated fibroblasts and fibrosis induces immunosuppression and accelerates pancreas cancer with reduced survival. Cancer Cell 2014:25:719-34.

9 Rhim AD, Oberstein PE, Thomas DH, et al. Stromal elements act to restrain, rather than support, pancreatic ductal adenocarcinoma. Cancer Cell 2014;25:735-47.

10 Jiang $H$, Liu $X$, Knolhoff BL, et al. Development of resistance to FAK inhibition in pancreatic cancer is linked to stromal depletion. Gut 2020;69:122-32.

11 Jiang $\mathrm{H}$, Hegde $\mathrm{S}$, Knolhoff $\mathrm{BL}$, et al. Targeting focal adhesion kinase renders pancreatic cancers responsive to checkpoint immunotherapy. Nat Med 2016;22:851-60. 